\title{
Clinical and next-generation sequencing findings in a Chinese family exhibiting severe familial exudative vitreoretinopathy
}

\author{
YING LIN $^{1 *}$, HONGBIN GAO $^{2,3 *}$, CHUAN CHEN $^{1,4^{*}}$, YI ZHU $^{1,4^{*}}$, TAO LI $^{1}$, BINGQIAN LIU $^{1}$, CHENGHONG MA $^{5}$ \\ HONGYE JIANG $^{6}$, YONGHAO LI ${ }^{1}$, YING HUANG ${ }^{1}$, QINGXIU WU ${ }^{1}$, HAICHUN LI $^{1}$, XIAOLING LIANG ${ }^{1}$, \\ CHENJIN JIN $^{1}$, JIANHUA YE ${ }^{5}$, XINHUA HUANG ${ }^{1}$ and LIN LU $^{1}$ \\ ${ }^{1}$ State Key Laboratory of Ophthalmology, Zhongshan Ophthalmic Center, Sun Yat-sen University, Guangzhou, \\ Guangdong 510060; ${ }^{2}$ Department of Toxicology, School of Public Health and Tropical Medicine, \\ Southern Medical University, Guangzhou, Guangdong 510515; ${ }^{3}$ Guangdong Laboratory Animals Monitoring Institute, \\ Key Laboratory of Guangdong Laboratory Animals, Guangzhou, Guangdong 510663, P.R. China; \\ ${ }^{4}$ Department of Molecular and Cellular Pharmacology, University of Miami Miller School of Medicine, \\ Miami, FL 33136, USA; ${ }^{5}$ Department of Endocrine, College of Clinical Medicine, \\ The First Affiliated Hospital of Guangdong Pharmaceutical University, Guangzhou, Guangdong 510080; \\ ${ }^{6}$ Department of Obstetrics and Gynecology, The First Affiliated Hospital, Sun Yat-sen University, \\ Guangzhou, Guangdong 510000, P.R. China
}

Received July 3, 2017; Accepted November 28, 2017

DOI: $10.3892 /$ ijmm.2017.3308

\begin{abstract}
Familial exudative vitreoretinopathy (FEVR) is a rare hereditary retinal disorder characterized by the premature arrest of vascularization in the peripheral retina. The aim of the present study was to characterize the clinical presentations of a Chinese family affected by bilateral severe FEVR, and to identify the underlying genetic variations. One family that presented with bilateral FEVR was recruited for this study. Comprehensive ophthalmic examinations, including best-corrected visual acuity, slit-lamp examination, fundus photography, fundus fluorescein angiography imaging and electroretinogram were performed. Genomic DNA was extracted from leukocytes of the peripheral blood collected from the affected and unaffected family members, as well as 200 unrelated control subjects from the same population. Next-generation sequencing of the candidate genes associated with ocular diseases was performed, and the identified mutations were validated by conventional polymerase chain
\end{abstract}

Correspondence to: Dr Lin Lu or Dr Xinhua Huang, State Key Laboratory of Ophthalmology, Zhongshan Ophthalmic Center, Sun Yat-sen University, 54 South Xianlie Road, Guangzhou, Guangdong 510060, P.R. China

E-mail: drlulinocular@126.com

E-mail: 1476557150@qq.com

${ }^{*}$ Contributed equally

Key words: familial exudative vitreoretinopathy, ATP binding cassette subfamily A member 4, LDL receptor related protein 5, mutation, next-generation sequencing reaction-based sequencing. The functional effects of the mutations were analyzed by polymorphism phenotyping (PolyPhen) and sorting intolerant from tolerant (SIFT). One heterozygous ATP binding cassette subfamily A member 4 (ABCA4) c.5693G $>$ A (p.R1898H) mutation in exon 40 and one heterozygous LDL receptor related protein 5 (LRP5) c.260T>G (p.I87S) mutation in exon 2 were identified in this family. To the best of our knowledge, the $A B C A 4$ c.5693G $>$ A (p.R1898H) mutation has not been reported in FEVR, and the $L R P 5$ c.260T $>\mathrm{G}$ (p.I87S) mutation is a novel mutation. PolyPhen and SIFT predicted that the amino acid substitution $\mathrm{R} 1898 \mathrm{H}$ in protein ABCA4 is benign, whereas the amino acid substitution I87S in protein LRP5 is damaging. A single nucleotide polymorphism c.266A>G (p.Q89R, rs41494349) was identified in exon 2 of LRP5. These findings expand the mutation spectrums of $A B C A 4$ and $L R P 5$, and will be valuable for genetic counseling and development of therapeutic interventions for patients with FEVR.

\section{Introduction}

Familial exudative vitreoretinopathy (FEVR; OMIM 133780, $305390,605750,601813$ and 613310) is a rare hereditary retinal disorder first reported by Criswick and Schepens in 1969 (1). It is characterized by the premature arrest of vascularization in the peripheral retina, resulting in peripheral retinal neovascularization, subretinal exudation, temporal radial retinal folds, macular dragging, and tractional retinal detachment (1-6). FEVR exhibits variable patterns of inheritance, including autosomal dominant (AD), autosomal recessive (AR), and $\mathrm{X}$-linked recessive $(7,8)$. The clinical manifestations of FEVR vary significantly depending on age; from very mild symptoms to complete blindness, even within the same family $(4,9)$. The diagnosis of FEVR is made based on the evidence of incomplete 
peripheral retinal vascularization in one or two eyes in patients born at pre-term or full-term, and must be differentiated from retinopathy of prematurity (ROP), Coats' disease, incontinentia pigmenti, and persistent fetal vasculature $(2,10,11)$. The clinical staging of FEVR is determined based on the presence of peripheral retinal avascularity (stage I), retinal neovascularization (stage II), extramacular retinal detachment (stage III), subtotal macula-involving retinal detachment (stage IV), and total retinal detachment (stage V) $(2,10)$. Treatment for FEVR is usually guided by the clinical stages. For the more advanced stages III-V, surgery is often required to remove scar tissue and release traction.

Currently, five genes have been identified to be mutated in FEVR, including low-density lipoprotein receptor-related protein 5 (LRP5; AD or AR; OMIM 603506), frizzled-4 (FZD4; AD or AR; OMIM 604579), tetraspanin-12 (TSPAN12; AD or AR; OMIM 613138), Norrie disease protein (NDP; X-linked; OMIM300658) and Zinc Finger Protein 408 (ZNF408; AD; OMIM 616454) (2). Among them, LRP5, FZD4, TSPAN12 and $N D P$ are all involved in the $\mathrm{Wnt} / \beta$-catenin signaling pathway, indicating the key regulatory role of this signaling pathway during retinal vascularization. In addition, the Wnt/ $\beta$-catenin signaling pathway communicates with other growth factor signaling pathways, such as fibroblast growth factor and transforming growth factor- $\beta$, which are critical for the regulation of cell growth and differentiation during development (12-19). A recent study by Salvo et al (20) sequenced 92 FEVR patients by next-generation sequencing (NGS), and identified mutations in LRP5, FZD4, TSPN12, NDP and ZNF408 that account for $19.6,15.2,8.7,6.5$ and $1.1 \%$ of the patients, respectively. Notably, the authors identified two patients with mutations in more than one disease-associated gene (20). However, the functional consequences of these mutations remain unclear.

As FEVR is a clinically heterogeneous disorder, molecular diagnosis provides useful information for disease diagnosis and genetic counseling. Furthermore, identification of genetic mutations in FEVR is the first stage of elucidating the pathogenesis of this disease. The aim of the present study was to characterize the clinical presentation of a family presenting with bilateral severe FEVR, and to identify the underlying genetic variations in this family.

\section{Materials and methods}

Study subjects and clinical examinations. A family presenting with bilateral FEVR was recruited for the present study. A total of five family members underwent complete ophthalmic examinations in the Zhongshan Ophthalmic Center, Sun Yat-sen University (Guangzhou, China). Visual acuity was examined using the early treatment diabetic retinopathy study chart (Precision Vision, Lasalle, IL, USA). Anterior segment photography was performed using the BX 900 Slit Lamp (Haag-Streit AG, Koeniz, Switzerland). Fundus photography and fundus fluorescein angiography (FFA) were performed using the Heidelberg Retina Angiograph (Heidelberg Engineering GmbH, Heidelberg, Germany) and the Ret Cam imaging system (Clarity Medical Systems, Inc., Pleasanton, CA, USA). The amplitudes of the rod and cone responses were assessed using the Espion electrophysiology system (Diagnosys LLC, Lowell, MA, USA) according to the electroretinogram (ERG) standards of the International Society for Clinical Electrophysiology of Vision (ISCEV; 2008 update). Physical examinations and B-scan ultrasonography were performed to confirm the diagnosis and exclude systemic diseases.

Target capture and NGS. A total volume of $15 \mathrm{ml}$ venous blood samples from the patient, the unaffected family members, and 200 unrelated control subjects from the same population were collected. Genomic DNA from peripheral blood leucocytes was extracted using the QIAamp DNABlood Midi kit (cat. no. 51104, Qiagen GmbH, Hilden, Germany) according to the manufacturer's instructions. The genomic DNA was fragmented by the Covaris LE220 Sonicator (Covaris, Woburn, MA, USA) to generate a paired-end library (200-250 bp). The library was enriched by array hybridization as previously described (21), followed by elution and post-capture amplification. The products were then subjected to the Agilent 2100 Bioanalyzer (Agilent Technologies, Inc., Santa Clara, CA, USA) and StepOnePlus Real-Time PCR System (Thermo Fisher Scientific, Inc., Waltham, MA, USA) for estimation of the enrichment magnitude. Subsequent to quality control, captured library sequencing was performed on the Illumina HiSeq2500 Analyzers (Illumina, Inc., San Diego, CA, USA) for 90 cycles per read to generate paired-end reads. Image analysis, error estimation and base calling were performed using the Illumina Pipeline software (version 1.3.4; Illumina, Inc.) to generate raw data (22).

A capture panel of inherited retinal-disease genes was previously designed and assessed in the present study. The capture panel comprised 708,919 bp that covered all exons together with the flanking exon and intron boundaries $( \pm 15 \mathrm{bp})$ of 175 genes, including 138 genes causing common inherited non-syndromic eye diseases and 54 genes causing syndromic eye diseases $(22,23)$.

Data analysis and mutation validation. To detect the potential variants in the family, bioinformatics processing and data analysis were performed after receiving the primary sequencing data. Previously published filtering criteria were used to generate 'clean reads' for further analysis (21). The 'clean reads' (with a length of $90 \mathrm{bp}$ ) derived from targeted sequencing and filtering were subsequently aligned to the human genome reference (hg19) using the Burrows Wheeler Aligner (BWA) Multi-Vision software package (version 0.7.12, http://bio-bwa.sourceforge.net) (24). Following alignment, the output files were used to perform sequencing coverage and analysis of the target region, single-nucleotidevariant (SNV), and insertion/deletion (indel) calling. SOAPsnp software (version 1.03, http://soap.genomics.org.cn/soapsnp.html) (24) and Samtools (version 1.6, http://samtools.sourceforge.net) (25) were used to detect SNVs and indels. All SNVs and indels were filtered and estimated via multiple databases, including the National Center for Biotechnology Information (NCBI) dbSNP (https://www.ncbi.nlm.nih.gov/snp/), HapMap1000 human genome dataset (http://www.internationalgenome.org/home), and a database of 200 Chinese healthy adults based on the blood samples of the 200 untreated control patients included in the present study. 
The identified mutations were validated using conventional polymerase chain reaction (PCR)-based sequencing methods. Exon 40 of the $A B C A 4$ gene (OMIM 601691) and the exon 2 of the LRP5 gene were amplified by PCR using the respective primers (Table I) as previously described (26-28). Briefly, the PCR reactions were conducted in a total volume of $50 \mu \mathrm{l}$ using the following thermal cycling profile: One cycle at $94^{\circ} \mathrm{C}$ for $5 \mathrm{~min}$, followed by 40 cycles at $94^{\circ} \mathrm{C}$ for $45 \mathrm{sec}, 60-62^{\circ} \mathrm{C}$ for $45 \mathrm{sec}, 72^{\circ} \mathrm{C}$ for $45 \mathrm{sec}$, and a final extension step at $72^{\circ} \mathrm{C}$ for $10 \mathrm{~min}$. The PCR products were sequenced from each direction with an ABI3730 Automated Sequencer (Thermo Fisher Scientific, Inc.). The sequencing results were analyzed using the SeqManII program of the Lasergene package (DNAStar, Inc., Madison, WI, USA) and compared with the reference sequences in the NCBI database $(29,30)$.

Interpretation of the genetic variants. To predict the effect of missense variants, polymorphism phenotyping (PolyPhen) and sorting intolerant from tolerant (SIFT) were used to predict the potential impact of a single amino acid substitution on the protein structure and function using straight forward physical and comparative considerations $(22,23,31)$. Variants were considered pathogenic when at least one of the two programs predicted deleterious effect of the amino acid substitution on the protein structure or function. The Human Gene Mutation Database was used to screen mutations reported in the published studies. In addition, HomoloGene (https://www.ncbi.nlm.nih. gov/homologene) was used to assess whether the mutated amino acid residues were conserved across different species.

All experimental protocols were performed according to the guidelines approved by the ethics committee of Zhongshan Ophthalmic Center. Written informed consent forms were obtained from all family members prior to the study. The tenets of the Declaration of Helsinki were followed throughout the study.

\section{Results}

Clinical manifestations. The family in the current study was from the southern area of China. The pedigree indicated that the FEVR was inherited in an AD manner (Fig. 1). The affected father (I:1) exhibited a normal anterior segment with transplant cornea and lens in each eye (Fig. 2A-D). Fundus photography and FFA exhibited peripheral retinal degeneration (Fig. 2E and F; black arrows) and brush-like peripheral vessels (Fig. 2G and $\mathrm{H}$; white arrows), which are considered typical signs of FEVR. The mother (I:2) and the 9-year-old daughter (II:1) presented with normal anterior segments and normal retinal appearance without leakage and degeneration (Figs. 3 and 4). The 7-year-old son (II:2; the proband) presented with local retinal detachment and low vision at birth. He exhibited bilateral nystagmus. Anterior-segment examination demonstrated local lens opacities in the right eye (Fig. 5A and B; white arrows), whereas the left eye was relatively normal (Fig. 5C and D). Fundus examination exhibited knife-like retinal fold (falciform retinal fold), macular dragging and retinal detachment (Fig. 5E and F; black arrows). FFA demonstrated peripheral retinal avascularity with abnormal vessels and leakage (Fig. 5G and H; white arrows). A B-scan indicated retinal detachment (Fig. 5I and J; white

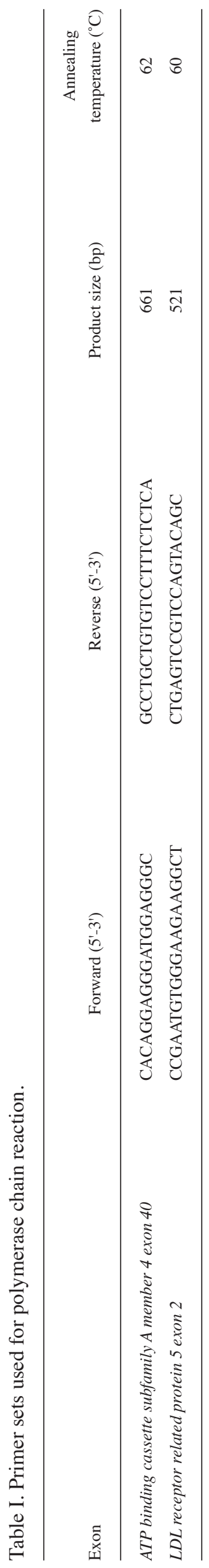


।

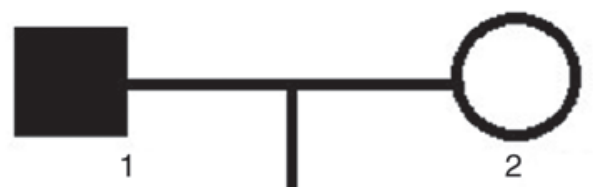

II

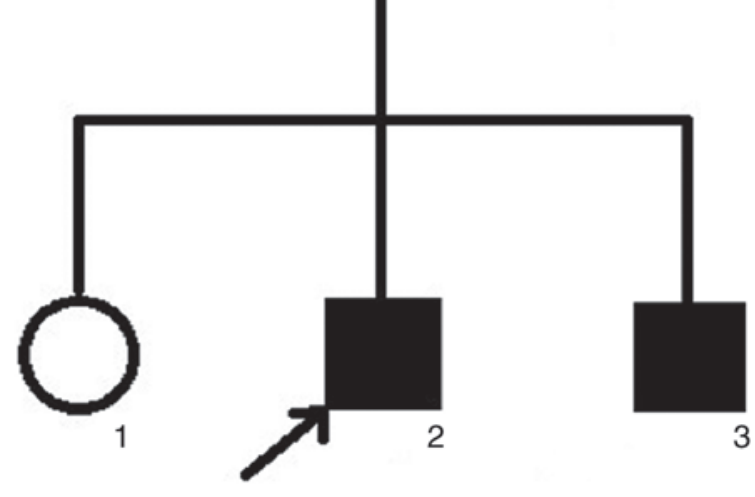

Figure 1. Pedigree of the Chinese family with FEVR. Squares denote males and circles denote females. The solid symbols indicate ophthalmologist-confirmed FEVR, and the open symbols indicate unaffected individuals. The arrow indicates the proband. FEVR, familial exudative vitreoretinopathy.

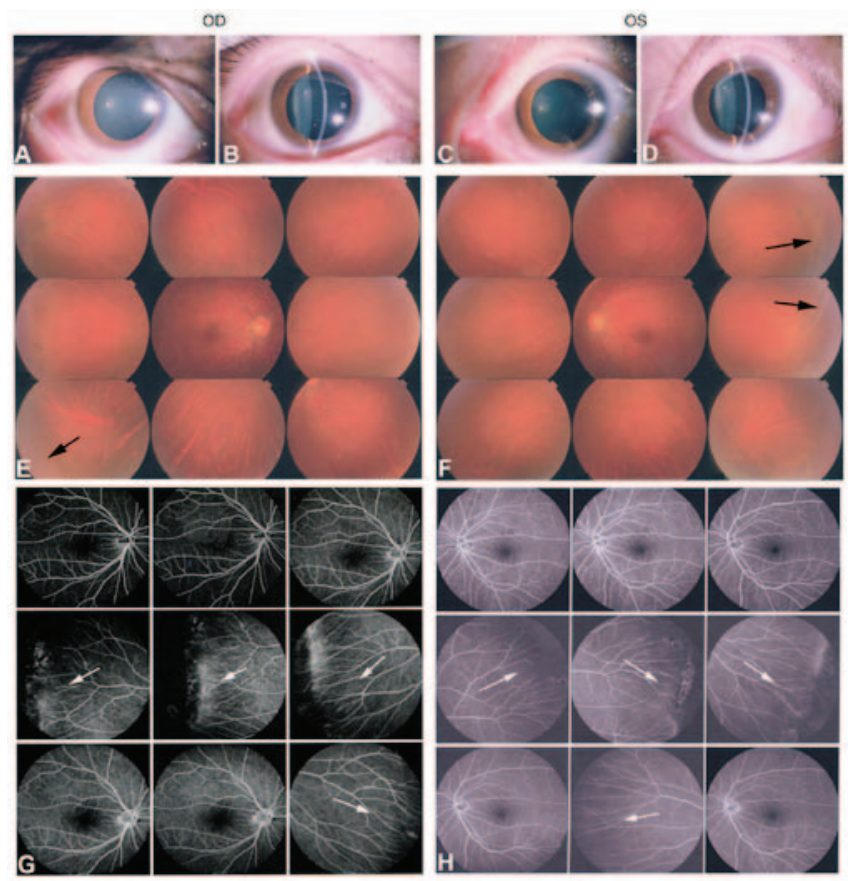

Figure 2. Clinical manifestations of the father (I:1). (A-D) Anterior segment photography demonstrates the transplant cornea and lens of each eye. (E and F) Fundus photography exhibits peripheral retinal degeneration (black arrows). (G and $\mathrm{H}$ ) Fluorescein angiography demonstrated brush-like peripheral vessels (white arrows). OD, right eye; OS, left eye.

arrows). ERG exhibited mild abnormal rod responses and severe abnormal cone responses (Fig. 6). Another affected 6-year-old son (II:3) presented with bilateral nystagmus, but normal anterior segment (Fig. 7A-D). Fundus examination revealed straight retinal blood vessels and the absence of a macular arch ring (Fig. 7E and F). The clinical manifestations of the family members are summarized in Table II.

Mutation screening and bioinformatics analysis of the mutations. One recurrent heterozygous $A B C A 4$ mutation c.5693G $>A$

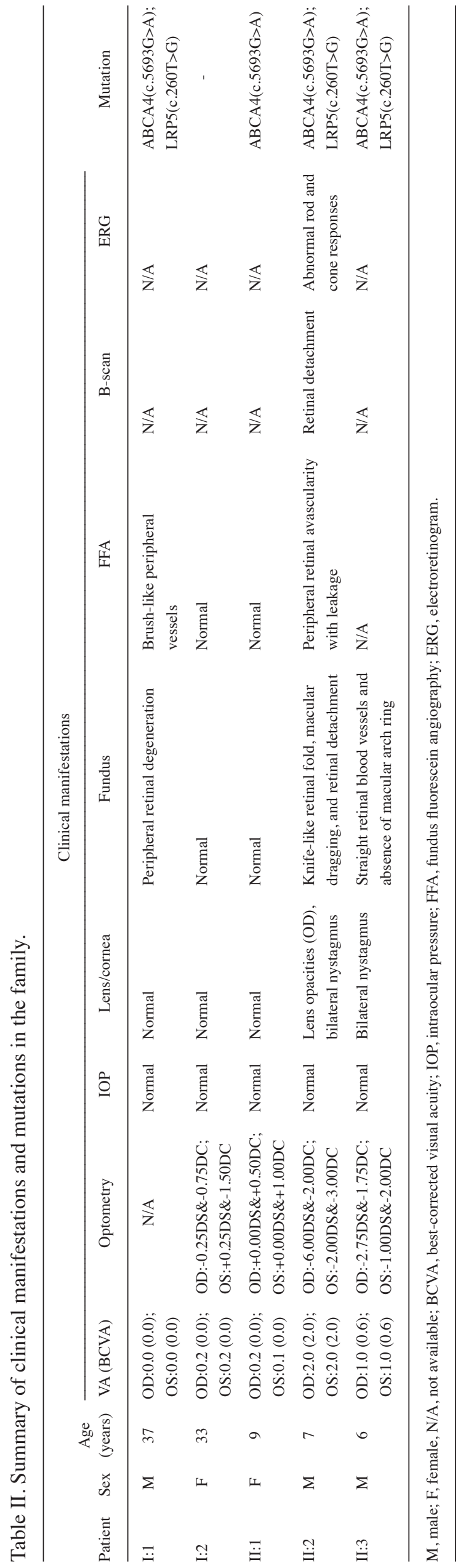



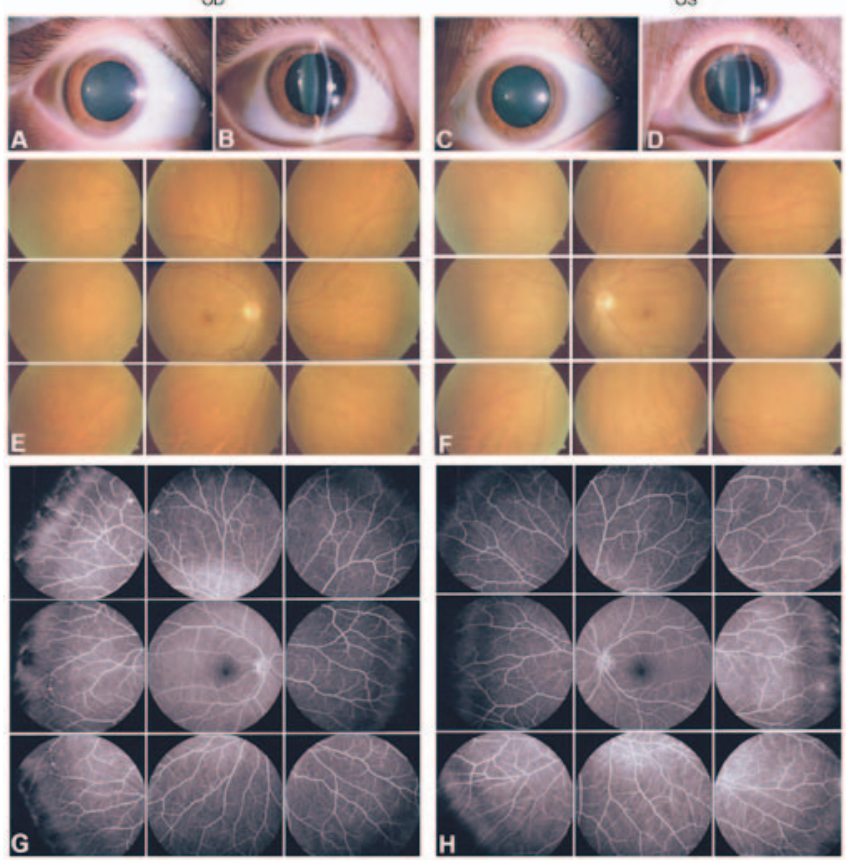

Figure 3. Clinical manifestations of the mother (I:2). (A-D) Anterior segment photography demonstrates the transplant cornea and lens of each eye. (E-H) Fundus photography and fluorescein angiography exhibited a normal retinal appearance without leakage or degeneration. OD, right eye; OS, left eye.
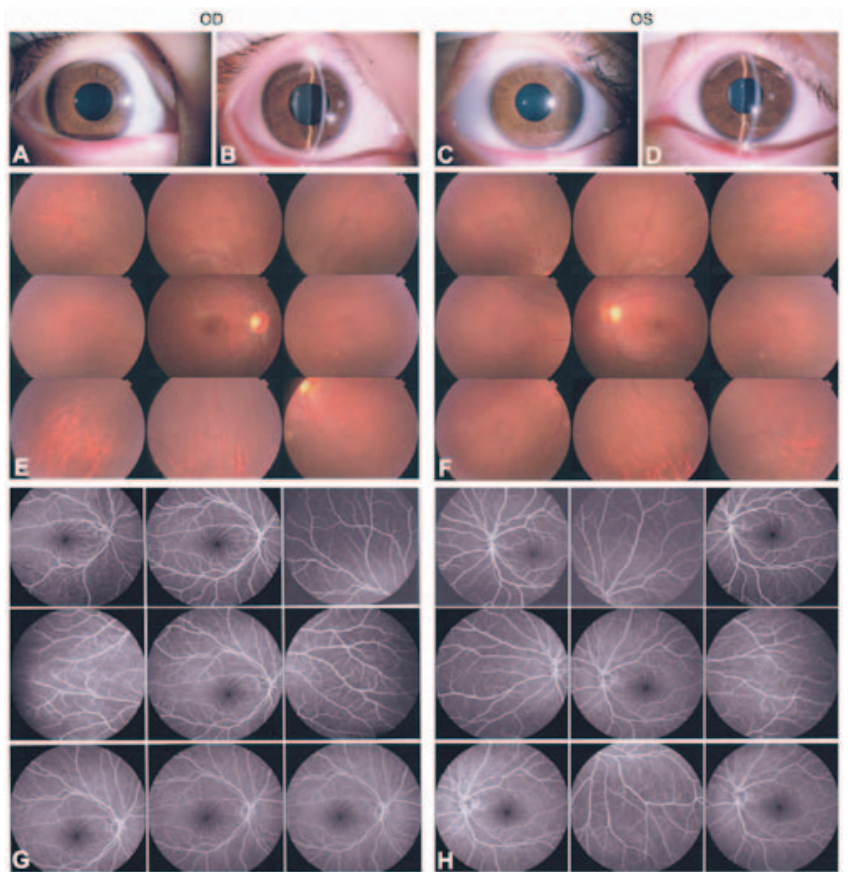

Figure 4. Clinical manifestations of the daughter (II:1). (A-D) Anterior segment photography demonstrates the transplant cornea and lens of each eye. (E-H) Fundus photography and fluorescein angiography exhibited normal retinal appearance without leakage or degeneration. OD, right eye; OS, left eye.

(p.R1898H) in exon 40 was identified in the cases of I:1, II:1, II:2 and II:3 (Fig. 8A and Table II). Multiple sequence alignment indicated that the residue at position 1898 of ABCA4 is not highly conserved (Fig. 8B). Polyphen (Fig. 8C) and SIFT predicted that the amino acid substitution $\mathrm{R} 1898 \mathrm{H}$ in the protein ABCA4 is benign.
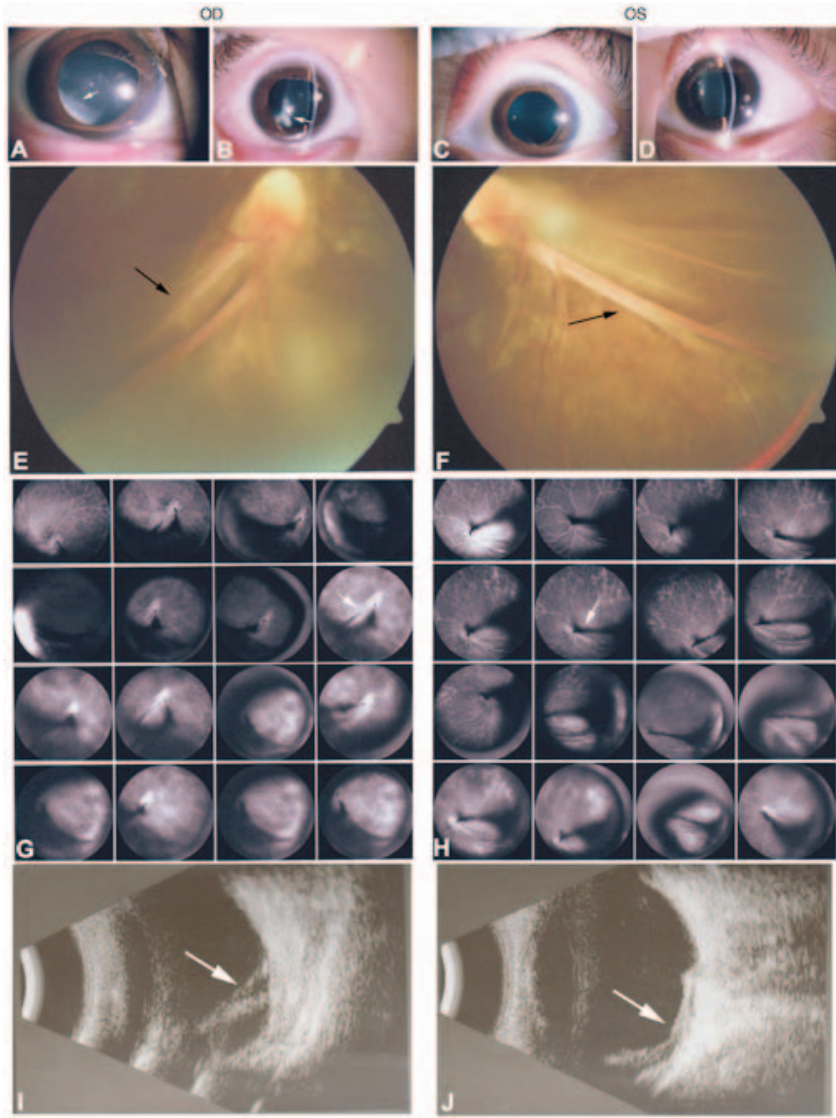

Figure 5. Clinical manifestations of the son (proband; II:2). (A-D) Anterior segment photography demonstrates the local lens opacities in (A and B; white arrows) in the right eye, whereas (C and D) the left eye was relatively normal. (E and F) Fundus photography exhibited knife-like retinal fold (falciform retinal fold), macula dragging, and optic discand retinal detachment (black arrows). ( $\mathrm{G}$ and $\mathrm{H}$ ) fluorescein angiography exhibited peripheral retinal avascularity with abnormal vessels and leakage (white arrows). (I and J) A B-scan demonstrated retinal detachment in each eye (white arrows). OD, right eye; OS, left eye.

One novel heterozygous LRP5 mutation c.260T>G (p.I87S) in exon 2 was identified in this family (I:1, II:2 and II:3; Fig. 9 and Table II). A single nucleotide polymorphism c.266A $>\mathrm{G}$ (p.Q89R, rs41494349) in exon 2 was identified in the cases of I:2, II:1 and II:3 (Fig. 9A) (13). The residues at position 87 and 89 of LRP5 are highly conserved across species (Fig. 9B). PolyPhen (Fig. 9C) and SIFT predicted that the amino acid substitution I87S in the protein LRP5 is damaging. PolyPhen (Fig. 9C) predicted that the amino acid substitution Q89R in the protein LRP5 is benign.

These two mutations [c.5693G $>$ A (p.R1898H) in $A B C A 4$ and c.260T $>\mathrm{G}$ (p.I87S) in LRP5] were not observed in the 200 unrelated subjects from the same population.

\section{Discussion}

FEVR exhibits highly variable expressivity in the affected individuals even in the same family, which may be due to the presence of genetic modifiers and environmental factors (1). For example, alterations of the oxygen levels in the intrauterine environment or exposure to certain drugs, may predispose the affected individuals to develop clinical symptoms (2). Additionally, FEVR presents asymmetrically, 
A

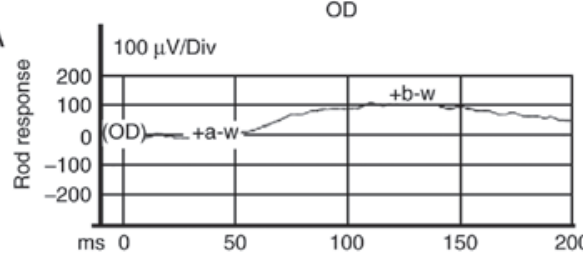

C

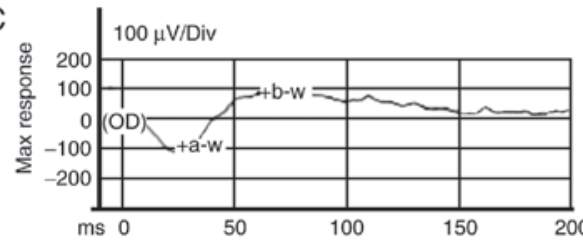

E

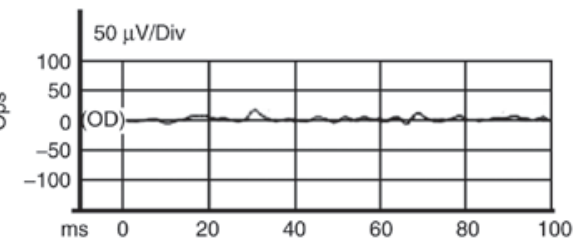

G

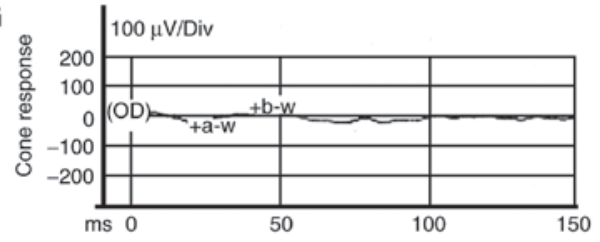

I

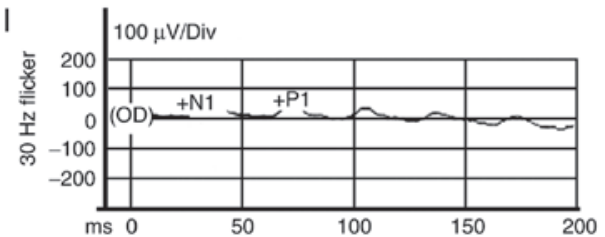

B

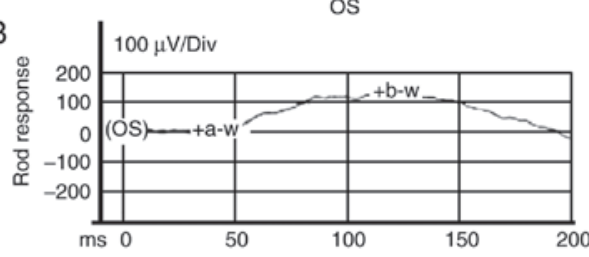

D

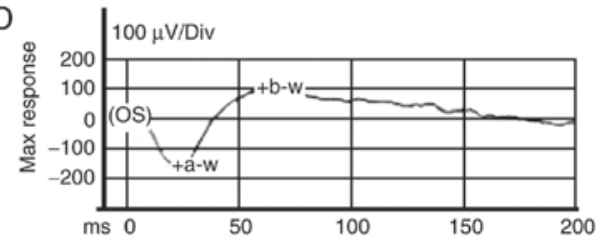

$\mathrm{F}$

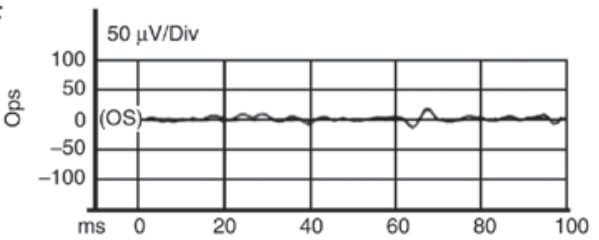

$\mathrm{H}$

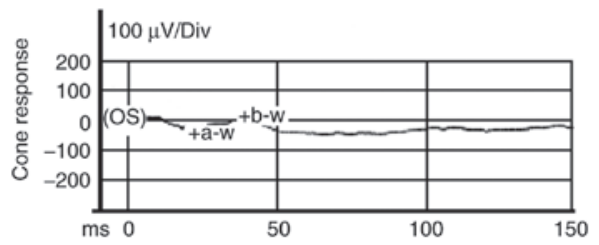

$J$

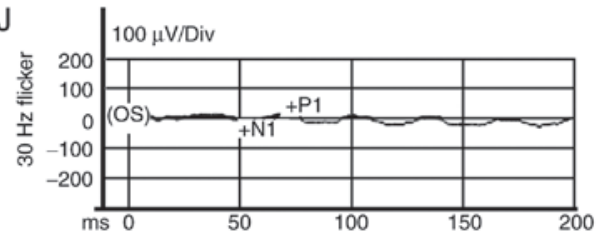

Figure 6. ERG examinations of the son (proband; II:2). (A-F) ISCEV standard scotopic ERG. (G-J) ISCEV standard photopic ERG. ERG indicated mild abnormal rod responses and severe abnormal cone responses. ERG, electroretinogram; ISCEV, International Society for Clinical Electrophysiology of Vision; a-w, a-wave; b-w, b-wave; Div, division; N1, first negative wave; P1, first positive wave; Ops, oscillatory potentials. OD, right eye; OS, left eye.

$\mathrm{OD}$

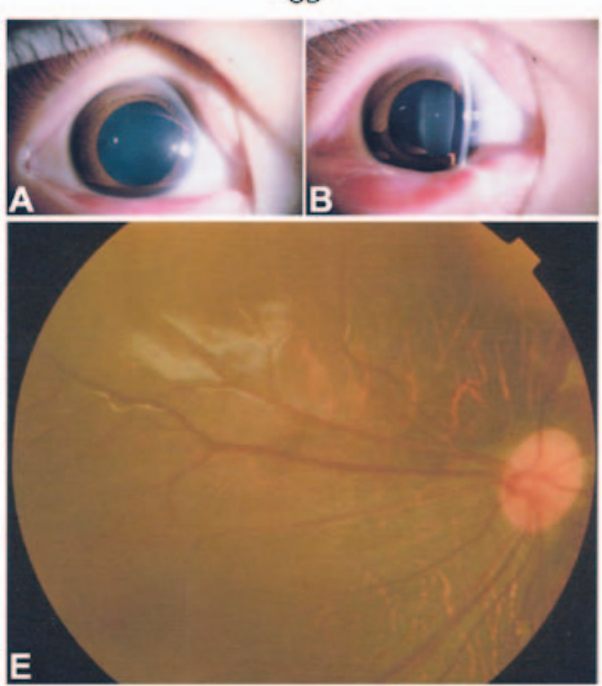

os

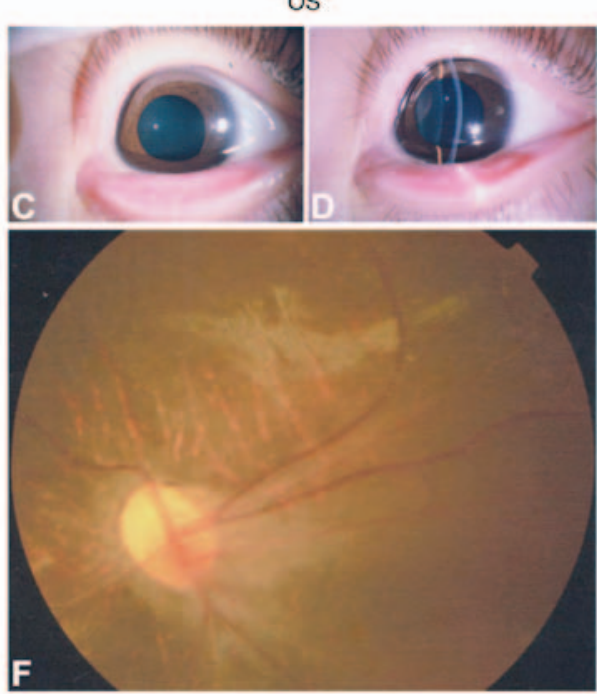

Figure 7. Clinical manifestations of the son (II:3). (A-D) Anterior segment photography demonstrated the transplant cornea and lens of each eye. (E and F) Fundus photography exhibited straight retinal blood vessels and absence of a macular arch ring. OD, right eye; OS, left eye.

where a single eye develops retinal detachment and the other eye exhibits no observable clinical signs, potentially due to local microenvironmental differences during retinal vasculogenesis (2). In the present study, the proband (II:2) 
A

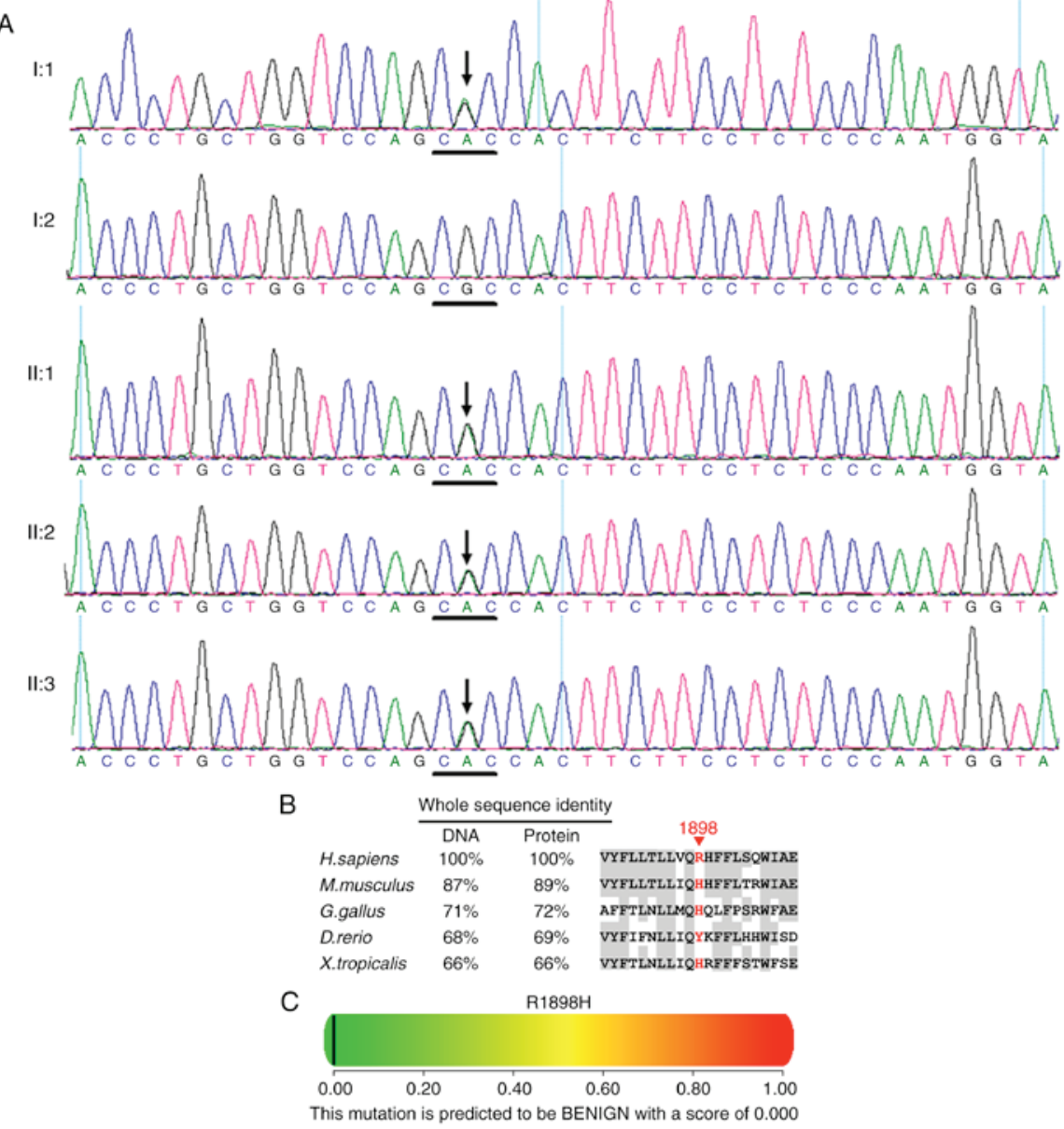

Figure 8. Identification of one recurrent heterozygous $A B C A 4$ mutation in the family members. (A) DNA sequence chromatograms of the unaffected and affected family members. One recurrent heterozygous ABCA4 mutation c.5693G>A (p.R1898H) in exon 40 was identified in the cases of I:1, II:1, II:2 and II:3. (B) Multiple-sequence alignment of the $A B C A 4$ genes and $\mathrm{ABCA} 4$ proteins from different species. The red triangle indicates the location of the mutation The Arg 1898 residue is not highly conserved across species. (C) Polymorphism phenotyping predicted that the amino acid substitution R1898H in the protein ABCA4 is benign. ABCA4, ATP binding cassette subfamily A member 4.

exhibited various bilateral retinal folds, macula dragging and retinal detachment, whereas the other individuals carrying the same mutations exhibited relatively mild symptoms (I:1 and II:3). The affected individuals (I:1, II:2 and II:3) presented with brush-like peripheral vessels or avascularity, which are considered as the hallmarks of FEVR $(32,33)$. The proband (II:2) exhibited decreased amplitudes and prolonged implicit times of the ERG components, which were previously reported in the FEVR patients (34). Notably, the FEVR patients in the present study did not have a history of prematurity, which is an important clinical feature that distinguishes FEVR from ROP, as the vitreoretinal manifestations of these two diseases are very similar (35).

In the present study, two mutations were identified in the affected individuals of the family. To the best of our knowledge, the $A B C A 4$ mutation c.5693G $>$ A (p.R1898H) has not been reported in FEVR. The $A B C A 4$ gene encodes a large glycoprotein ABCA4 with 2,273 amino acids. The ABCA4 protein is composed of two structurally-associated tandem-arranged halves. The $\mathrm{N}$ and $\mathrm{C}$ halves are predicted to have a single membrane-spanning segment followed by a large exocytoplasmic domain, a transmembrane domain (TMD) and a nucleotide-binding domain (36,37). A highly conserved VFVNFA motif near the C-terminus is essential for the cholesterol efflux and apolipoprotein A-I binding activities of ABCA4 (Fig. 10A). Mutations in the $A B C A 4$ gene are responsible for a large variety of retinal degenerative diseases, including Stargardt disease, cone-rod degeneration, retinitis pigmentosa, and age-related macular degeneration $(38,39)$. The mutation c.5693G $>$ A (p.R1898H) identified in the present study alters the amino acid residue close to the TMD (Fig. 10A) (39). Two unrelated pedigrees with Stargardt disease have been reported to carry this mutation $(6,40-42)$. However, bioinformatic analyses by PolyPhen and SIFT predicted that this mutation is not harmful (Fig. 8C) and the residue Arg 1898 on ABCA4 is not conserved across species (Fig. 8B). Therefore, detailed studies are required to determine the functional consequences of this mutation. Additionally, this mutation is associated with age-related macular degeneration (AMD) $(6,40,41)$. The present study was unable to exclude the possibility that the individuals carrying this mutation in this family are predisposed to the development of AMD; therefore, follow-up studies are required.

The LRP5 mutation c.260T>G (p.I87S) in exon 2 identified in this family is a novel mutation. Compared with FZD4, TSPN12, NDP and ZNF408, LRP5 is most frequently mutated in FEVR, likely due to its large coding region $(22,43-45)$. LRP5 and its homologue LRP6 encode single-span transmem- 


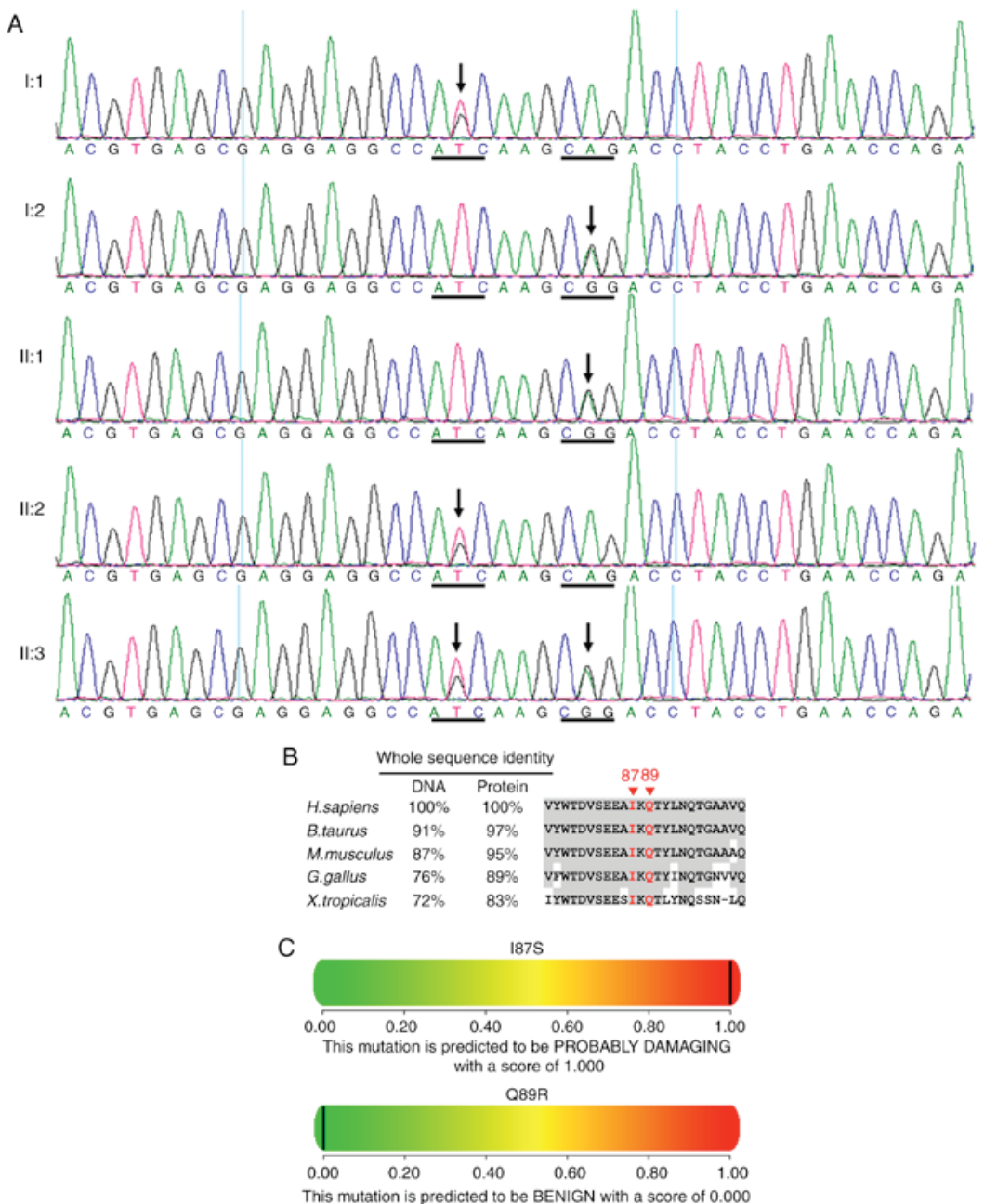

Figure 9. Identification of a novel heterozygous LRP5 mutation and one SNP in the family members. (A) DNA sequence chromatograms of the unaffected and affected family members. One heterozygous $L R P 5$ mutation c.260T $>\mathrm{G}$ (p.I87S) in exon 2 was identified in the cases of I:1, II:2 and II:3. One SNP c.266A>G (p.Q89R) in exon 2 was identified in the cases of I:2, II:1 and II:3. (B) Multiple-sequence alignment of the LRP5 genes and LRP5 proteins from different species. The red triangles indicate the position of the mutation and the SNP. The two amino acid residues are conserved across species. (C) Polymorphism phenotyping predicted that the amino acid substitution I87S in the protein LRP5 is damaging, while the amino acid substitution Q89R is benign. LRP5, LDL receptor related protein 5; SNP, single nucleotide polymorphism.
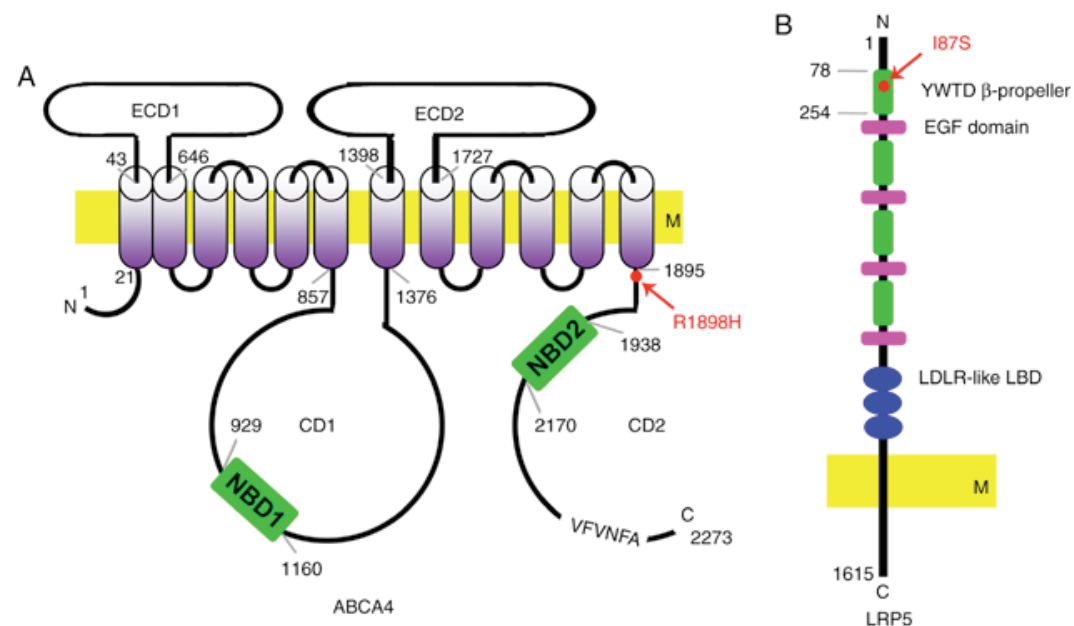

Figure 10. Schematic diagrams of the ABCA4 and LRP5 protein indicating the location of the mutations. (A) Predicted domains of ABCA4. ABCA4 protein consists of 2,273 amino acids. Its structure contains two ECDs (ECD1 and ECD2), two CDs (CD1 and CD2), 12 transmembrane segments, and a VFVNFA motif. Two NBDs (NBD1 and NBD2) are in the CD1 and CD2, respectively. The p.R1898H mutation is close to the last transmembrane segment (red arrow). (B) Predicted domains of LRP5. The LRP5 protein consists of 1,615 amino acids. Its extracellular segment contains four YWTD $\beta$-propeller domains and EGF-like domains. These domains are followed by three LDLR-like ligand-binding domains. The mutation p.I87S is in the first YWTD $\beta$-propeller domain. ABCA4, ATP binding cassette subfamily A member 4; LRP5, LDL receptor related protein 5; ECD, exocytoplasmic domains; CD, cytoplasmic domain; NBD, nucleotide binding domains; EGF, epidermal growth factor; LDLR, low density lipoproteins receptor; M, membrane. 
brane receptors, LRP5/LRP6 that interact with the seven-pass transmembrane receptor, FZD4 to bind Wnt, activating the canonical Wnt- $\beta$-catenin signaling pathway $(46,47)$. Norrin, a protein encoded by $N D P$, acts as a ligand and interacts with the FZD4-LRP5 complex, stabilizing the cytoplasmic $\beta$-catenin (48). Additionally, Norrin cooperates with TSPAN12 to promote multimerization of FZD4 (49). Although the binding sites of LRP5 to FZD4 and Norrin have not been fully elucidated, it has been proposed via comparative modeling that the missense mutations in LRP5 may disrupt the protein-binding sites $(15,50)$. The I87S mutation in LRP5 is in the first YWTD $\beta$-propeller domain (Figs. 9B and 10B). This domain is responsible for the binding of LRP5/LRP6 to the extracellular ligands. Therefore, this mutation may compromise the Wnt- $\beta$-catenin signaling, which is essential for retinal vascular development.

In conclusion, a detailed characterization of one Chinese family with bilateral FEVR was performed, and two trans heterozygous mutations were identified in ABCA4 and LRP5. These findings expand the mutation spectrums of $A B C A 4$ and $L R P 5$, and will be valuable for genetic counseling and development of therapeutic interventions for FEVR patients. Although our understanding of the function of ABCA4 and LRP5 proteins remains limited, the discovery of these mutant variants provides an opportunity and rationale for in-depth mechanistic studies, and may help to reveal the critical physiology underlying associated retinal development disorders in general.

\section{Acknowledgements}

The authors would like to thank the patients, their families and the control volunteers for participating in the present study. The study was supported by the National Natural Science Foundation of China (grant nos. 81500709, 81570862 and 81670872) and the State Scholarship Fund from the China Scholarship Council.

\section{References}

1. Criswick V and Schepens C: Familial exudative vitreoretinopathy. Am J Ophthalmol 68: 578-594, 1969.

2. Gilmour D: Familial exudative vitreoretinopathy and related retinopathies. Eye 29: 1-14, 2015.

3. Riveiro-Alvarez R, Trujillo-Tiebas MJ, Gimenez-Pardo A, Garcia-Hoyos M, Cantalapiedra D, Lorda-Sanchez I, Rodríguez de Alba M, Ramos C and Ayuso C: Genotype-phenotype variations in five Spanish families with Norrie disease or X-linked FEVR. Mol Vis 11: 705-712, 2005.

4. Sizmaz S, Yonekawa Y and Trese MT: Familial exudative vitreoretinopathy. Turk J Ophthalmol 45: 164-168, 2015.

5. Khwarg JW, Bourla D, Gonzales CA and Schwartz SD: Familial exudative vitreoretinopathy and macular hole exhibited in same individual. Semin Ophthalmol 22: 85-86, 2007.

6. Lewis RA, Shroyer NF, Singh N, Allikmets R, Hutchinson A, Li Y, Lupski JR, Leppert M and Dean M: Genotype/phenotype analysis of a photoreceptor-specific ATP-binding cassette transporter gene, ABCR, in Stargardt disease. Am J Hum Gene 64: 422-434, 1999.

7. Tang M, Ding X, Li J, Hu A, Yuan M, Yang Y, Zhan Z, Li Z and Lu L: Novel mutations in FZD4 and phenotype-genotype correlation in Chinese patients with familial exudative vitreoretinopathy. Mol Vis 22: 917-932, 2016

8. Li JK, Fei P, Li Y, Huang QJ, Zhang Q, Zhang X, Rao YQ, Li J and Zhao P: Identification of novel KIF11 mutations in patients with familial exudative vitreoretinopathy and a phenotypic analysis. Sci Rep 6: 26564, 2016.

9. Benson WE: Familial exudative vitreoretinopathy. Trans Am Ophthalmol Soc 93: 473-521, 1995.
10. Ranchod TM, Ho LY, Drenser KA, Capone A and Trese MT: Clinical presentation of familial exudative vitreoretinopathy. Ophthalmology 118: 2070-2075, 2011.

11. Kondo H, Kusaka S, Yoshinaga A, Uchio E, Tawara A and Tahira T: Genetic variants of FZD4 and LRP5 genes in patients with advanced retinopathy of prematurity. Mol Vis 19: 476: 485, 2013.

12. Buchtova M, Oralova V, Aklian A, Masek J, Vesela I, Ouyang Z, Obadalova T, Konecna Z, Spoustova T, Pospisilova T, et al: Fibroblast growth factor and canonical WNT/ $\beta$-catenin signaling cooperate in suppression of chondrocyte differentiation in experimental models of FGFR signaling in cartilage. Biochim Biophys Acta 1852: 839-850, 2015.

13. Hiyama A, Sakai D, Tanaka M, Arai F, Nakajima D, Abe K and Mochida J: The relationship between the Wnt/ $\beta$-catenin and TGF- $\beta$ /BMP signals in the intervertebral disc cell. J Cell Physiol 226: 1139-1148, 2011.

14. Lu GQ, Wu ZB, Chu XY, Bi ZG and Fan WX: An investigation of crosstalk between Wnt/ $\beta$-catenin and transforming growth factor- $\beta$ signaling in androgenetic alopecia. Medicine (Baltimore) 95: e4297, 2016.

15. Fujimura $\mathrm{N}: \mathrm{WNT} / \beta$-Catenin signaling in vertebrate eye development. Front Cell Develop Biol 4: 138, 2016.

16. Zhang Y, Morgan R, Chen C, Cai Y, Clark E, Khan WN, Shin SU, Cho HM, Al Bayati A, Pimentel A and Rosenblatt JD: Mammary-tumor-educated B cells acquire LAP/TGF-beta and PD-L1 expression and suppress anti-tumor immune responses. Int Immunol 28: 423-433, 2016.

17. Tan X, Zhu Y, Chen C, Chen X, Qin Y, Qu B, Luo L, Lin H, Wu M, Chen W and Liu Y: Sprouty2 suppresses epithelial-mesenchymal transition of human lens epithelial cells through blockade of Smad2 and ERK1/2 pathways. PLoS One 11: e0159275, 2016.

18. Qin Y, Zhu Y, Luo F, Chen C, Chen X and Wu M: Killing two birds with one stone: Dual blockade of integrin and FGF signaling through targeting syndecan- 4 in postoperative capsular opacification. Cell Death Dis 8: e2920, 2017.

19. Tan X, Chen C, Zhu Y, Deng J, Qiu X, Huang S, Shang F, Cheng B and Liu Y: Proteotoxic stress desensitizes TGF-beta signaling through receptor downregulation in retinal pigment epithelial cells. Curr Mol Med 17: 189-199, 2017.

20. Salvo J, Lyubasyuk V, Xu M, Wang H, Wang F, Nguyen D, Wang K, Luo H, Wen C, Shi C, et al: Next-generation sequencing and novel variant determination in a cohort of 92 familial exudative vitreoretinopathy patients. Invest Ophthalmol Vis Sci 56: 1937-1946, 2015.

21. Wei X, Ju X, Yi X, Zhu Q, Qu N, Liu T, Chen Y, Jiang H, Yang G, Zhen R, et al: Identification of sequence variants in genetic disease-causing genes using targeted next-generation sequencing. PLoS One 6: e29500, 2011.

22. Li T, Lin Y, Gao H, Chen C, Zhu Y, Liu B, Lian Y, Li Y, Zhou W, Jiang H, et al: Two heterozygous mutations identified in one Chinese patient with bilateral macular coloboma. Mol Med Rep 16: 2505-2510, 2017.

23. Avila-Fernandez A,Perez-Carro R, Corton M, Lopez-Molina MI, Campello L, Garanto A, Fernandez-Sanchez L, Duijkers L, Lopez-Martinez MA, Riveiro-Alvarez R, et al: Whole-exome sequencing reveals ZNF408 as a new gene associated with autosomal recessive retinitis pigmentosa with vitreal alterations. Hum Mol Genet 24: 4037-4048, 2015.

24. Li R, Li Y, Fang X, Yang H, Wang J, Kristiansen K and Wang J: SNP detection for massively parallel whole-genome resequencing. Genome Res 19: 1124-1132, 2009.

25. Li H, Handsaker B, Wysoker A, Fennell T, Ruan J, Homer N, Marth G, Abecasis G and Durbin R; 1000 Genome Project Data Processing Subgroup: The sequence alignment/map format and SAMtools. Bioinformatics 25: 2078-2079, 2009.

26. Lin Y, Ai S, Chen C, Liu X, Luo L, Ye S, Liang X, Zhu Y, Yang H and Liu Y: Ala344 Pro mutation in the FGFR2 gene and related clinical findings in one Chinese family with Crouzon syndrome. Mol Vis 18: 1278-1282, 2012.

27. Lin Y, Liang X, Ai S, Chen C, Liu X, Luo L, Ye S, Li B, Liu Y and Yang H: FGFR2 molecular analysis and related clinical findings in one Chinese family with Crouzon syndrome. Mol Vis 18: 449-454, 2012.

28. Lin Y, Liu X, Yu S, Luo L, Liang X, Wang Z, Chen C, Zhu Y, Ye S, Yan $\mathrm{H}$ and Liu Y: PAX6 analysis of two sporadic patients from southern China with classic aniridia. Mol Vis 18: 2190-2194, 2012.

29. Lin Y, Li T, Gao H, Lian Y, Chen C, Zhu Y, Li Y, Liu B, Zhou W, Jiang H, et al: Bestrophin 1 gene analysis and associated clinical findings in a Chinese patient with Best vitelliform macular dystrophy. Mol Med Rep 16: 4751-4755, 2017. 
30. Lin Y, Gao H, Ai S, Eswarakumar JV, Chen C, Zhu Y, Li T, Liu B, Liu X, Luo L, et al: C278F mutation in FGFR2 gene causes two different types of syndromic craniosynostosis in two Chinese patients. Mol Med Rep 16: 5333-5337, 2017.

31. Nikopoulos K, Gilissen C, Hoischen A, van Nouhuys CE, Boonstra FN, Blokland EA, Arts P, Wieskamp N, Strom TM, Ayuso C, et al: Next-generation sequencing of a $40 \mathrm{Mb}$ linkage interval reveals TSPAN12 mutations in patients with familial exudative vitreoretinopathy. Am J Hum Genet 86: 240-247, 2010

32. Miyakubo H, Hashimoto $\mathrm{K}$ and Miyakubo S: Retinal vascular pattern in familial exudative vitreoretinopathy. Ophthalmology 91: 1524-1530, 1984.

33. van Nouhuys CE: Signs, complications, and platelet aggregation in familial exudative vitreoretinopathy. Am J Ophthalmol 111: 34-41, 1991.

34. Yaguchi Y, Katagiri S, Fukushima Y, Yokoi T, Nishina S, Kondo $M$ and Azuma N: Electroretinographic effects of retinal dragging and retinal folds in eyes with familial exudative vitreoretinopathy. Sci Rep 6: 30523, 2016.

35. Dickinson JL, Sale MM, Passmore A, FitzGerald LM, Wheatley CM, Burdon KP, Craig JE, Tengtrisorn S, Carden SM, Maclean H and Mackey DA: Mutations in the NDP gene: Contribution to Norrie disease, familial exudative vitreoretinopathy and retinopathy of prematurity. Clin Exp Ophthalmol 34: 682-688, 2006

36. Shroyer NF, Lewis RA, Allikmets R, Singh N, Dean M, Leppert $\mathrm{M}$ and Lupski JR: The rod photoreceptor ATP-binding cassette transporter gene, ABCR, and retinal disease: From monogenic to multifactorial. Vision Res 39: 2537-2544, 1999.

37. Molday RS and Zhang K: Defective lipid transport and biosynthesis in recessive and dominant Stargardt macular degeneration. Prog Lipid Res 49: 476-492, 2010.

38. Koenekoop RK: The gene for Stargardt disease, ABCA4, is a major retinal gene: A mini-review. Ophthalmic Genet 24: 75-80, 2003.

39. Sun H, Smallwood PM and Nathans J: Biochemical defects in ABCR protein variants associated with human retinopathies. Nat Genet 26: 242-246, 2000.

40. Shroyer NF, Lewis RA and Lupski JR: Complex inheritance of ABCR mutations in Stargardt disease: Linkage disequilibrium, complex alleles, and pseudodominance. Hum Genet 106 : 244-248, 2000

41. Rivera A, White K, Stöhr H, Steiner K, Hemmrich N, Grimm T, Jurklies B, Lorenz B, Scholl HP, Apfelstedt-Sylla E and Weber BH: A comprehensive survey of sequence variation in the ABCA4 (ABCR) gene in Stargardt disease and age-related macular degeneration. Am J Hum Genet 67: 800-813, 2000.
42. Molday RS: Photoreceptor membrane proteins, phototransduction, and retinal degenerative diseases. The Friedenwald Lecture. Invest Ophthalmol Vis Sci 39: 2491-2513, 1998.

43. Pefkianaki M, Hasanreisoglu M, Suchy SF and Shields CL: Familial exudative vitreoretinopathy with a novel LRP5 mutation. J Pediat Ophthalmol Strabismus 53: e39-e42, 2016.

44. Musada GR, Syed H, Jalali S, Chakrabarti S and Kaur I: Mutation spectrum of the FZD-4, TSPAN12 AND ZNF408 genes in Indian FEVR patients. BMC Ophthalmol 16: 90, 2016

45. Yang H, Li S, Xiao X, Wang P, Guo X and Zhang Q: Identification of FZD4 and LRP5 mutations in 11 of 49 families with familial exudative vitreoretinopathy. Mol Vis 18: 2438-2446, 2012.

46. Tamai K, Semenov M, Kato Y, Spokony R, Liu C, Katsuyama Y, Hess F, Saint-Jeannet JP and He X: LDL-receptor-related proteins in Wnt signal transduction. Nature 407: 530-535, 2000.

47. Pinson KI, Brennan J, Monkley S, Avery BJ and Skarnes WC: An LDL-receptor-related protein mediates Wnt signalling in mice. Nature 407: 535-538, 2000.

48. Xu Q, Wang Y, Dabdoub A, Smallwood PM, Williams J, Woods C, Kelley MW, Jiang L, Tasman W, Zhang K and Nathans J: Vascular development in the retina and inner ear: Control by Norrin and Frizzled-4, a high-affinity ligand-receptor pair. Cell 116: 883-895, 2004

49. Junge HJ, Yang S, Burton JB, Paes K, Shu X, French DM, Costa M, Rice DS and Ye W: TSPAN12 regulates retinal vascular development by promoting Norrin-but not Wnt-induced FZD4/beta-catenin signaling. Cell 139: 299-311, 2009.

50. Toomes C, Bottomley HM, Jackson RM, Towns KV, Scott S, Mackey DA, Craig JE, Jiang L, Yang Z, Trembath R, et al: Mutations in LRP5 or FZD4 underlie the common familial exudative vitreoretinopathy locus on chromosome 11q. Am J Hum Genet 74: 721-730, 2004.

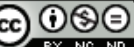

This work is licensed under a Creative Commons Attribution-NonCommercial-NoDerivatives 4.0 International (CC BY-NC-ND 4.0) License. 